this problem in class support this interpretation. They report that it is sometimes embarrassingly difficult to draw the solution, even through they know the principle involved. Similarly, the Es who administered the problem to the Ss in this study reported that it took them some time before they could rapidly draw the solution when demonstrating it to Ss.

\title{
Rigidity as a function of task complexity
}

RONALD RAY SCHMECK, Southern Illinois University, Carbondale, Ill. 62901, and FRED RIBICH, The Johns Hopkins Hospital, Baltimore, Md. 21205

Four groups of Ss were tested on hidden-word tasks consisting of 12 set-inducing items followed by 13 test items. One group, the simple-task group, received 7-letter items having a 4-letter word embedded in each. The other group, the complex-task group, received 10-letter items with each again having a 4-letter word embedded in it. The "set" response consisted of choosing alternate letters that formed a word, while the direct solution consisted of simply choosing 4 consecutive letters that formed a word. Direct solutions were possible only on the 13 test items. It was found that the complex experimental group produced significantly more direct solutions ("broke" the set more often) than did the simple experimental group. Results supported a behavioral model of rigidity based on the principles of Hull-Spence behavior theory.

To the present time, the literature contains no studies designed to investigate the interaction of task complexity and behavioral rigidity or mental set (see Luchins, 1959). Within the Hull-Spence system (Spence, 1956), a simple task is assumed to be one that involves few competing responses, with each of the competing responses having habit strengths that are significantly less than the habit strength of the dominant correct response. On the other hand, a complex task would be one having many competing responses with habit strengths that are almost equal. If it is assumed that behavioral variability is a function of the number and strengths of the competing responses possible within a particular situation, then it can be predicted that an $S$ will be more likely to "break" an established mental set while performing a complex task than while performing a simple task. The following study is designed to test this prediction using hidden-word tasks of varying complexity.

\section{SUBJECTS AND PROCEDURE}

The Ss were college freshmen enrolled in beginning psychology courses at Ohio University. They were tested in two groups of 30 each. One group received a simple list of hidden-word items, the other received a complex list. The simple list contained 25 items, each consisting of 7 letters. Ss were instructed to find a hidden 4-letter word without transposing the order of the letters. The first 12 items ("set-inducing" iter(s) could be solved, by choosing every other letter, beginning with the first letter. The last 13 items ("test" items) were solvable not only by the set method, but also by the more direct method of detecting a word composed of 4 consecutive letters embedded in the anagram. The complex list also contained 25 items, but in this case, each item was composed of 10 letters. Ss were given the same instructions as those given in the simple list. The first 12 items, again the set-inducing items, were solvable by the same alternating method as that used in the simple list, but the 1st letter of the alternating sequence varied from the first to the fourth position in the group of 10 letters. The remaining 13 items were again solvable both by the set method and by a more direct method like that of the simple list. The items were typed in capital letters, with 5 items to a page. Ss indicated their choice of letters by filling in a circle below each letter. Ss were also told not to go on to other items unless the previous item had been completed.

Two groups of five Ss each served as control groups. One group was given only the 13 test items from the simple list, while the other group was tested on only the 13 test items from the complex list. They were given the same instructions as the experimental groups.

\section{RESULTS AND DISCUSSION}

Several Ss in each of the two experimental groups failed to utilize the set method in solving the first 12 problems. Some produced nonsense words, and others skipped items. Thus, to insure that a set had indeed been formed, the following criteria were used to select those tests to be scored: (1) At least the eight items (Nos. 5-12) immediately preceding the test items were all solved by the set method, or (2) no more than three items were solved by a nonset method with none of these items being the last two of the set-inducing items (No. 11 and No. 12). Fourteen Ss in each of the two experimental groups met the criteria.

The control group produced $100 \%$ direct solutions of the 13 test items. The difference between the means of the complex experimental group (9.8) and the complex control group (13.0) was significant $[t(17)=3.68, p<.01]$. Also, the difference between the means of the simple experimental group (5.3) and the simple control group (13.0) was significant $[\mathrm{t}(17)=8.85, \mathrm{p}<.001]$. These significant differences indicate that the set-inducing items did, indeed, produce a set in both of the experimental groups.

The difference between the means of the simple and complex experimental grcups was highly significant $[\mathrm{t}(26)=3.60$, $\mathrm{p}<.01]$, with the complex group demonstrating more direct (nonset) solutions to the test items. Simply by varying the beginning position of the altemating sequence and by increasing the number of letters in the items, it was possible to produce a significant increase in set-breaking behavior. This increase is attributable to the increased behavioral variability resulting from the presence of more and stronger competing responses on the complex task.

REFERENCES

LUCHINS, A. S. Rigidity of behavior. Eugene: University of Oregon Books, 1959.

SPENCE, K. W. Behavior theory and conditioning. New Haven: Yale University Press, 1956. 